\section{Auto-avaliação de saúde bucal em idosos: análise com base em modelo multidimensional}

\author{
Objective and subjective factors related to \\ self-rated oral health among the elderly
}

\author{
${ }_{1}$ Curso de Odontologia, \\ Universidade Estadual \\ de Montes Claros, Montes \\ Claros, Brasil. \\ 2 Curso de Odontologia, \\ Faculdades Unidas do Norte \\ de Minas, Montes Claros, \\ Brasil. \\ ${ }^{3}$ Faculdade de Medicina, \\ Universidade Federal de \\ Minas Gerais, Belo Horizonte, \\ Brasil. \\ 4 Faculdade de Odontologia, \\ Universidade Federal de \\ Minas Gerais, Belo Horizonte, \\ Brasil. \\ Correspondência \\ A. M. E. B. L. Martins \\ Curso de Odontologia \\ Universidade Estadual de \\ Montes Claros. \\ Av. Cula Mangabeira 210 \\ Montes Claros, $M G$ \\ 39401-001, Brasil. \\ martins.andreamebl@gmail.com
}

\begin{abstract}
The aim of this study was to investigate factors associated with negative self-rated oral health. Elderly subjects from the Brazilian Ministry of Health's oral health survey (2002-2003) who rated their own oral health as bad or very bad were compared to those who rated their oral health as excellent, good, or fair, using prevalence ratios based on Poisson regression. The minority (870; 17\%) gave their own oral health a negative rating. Negative self-rated oral health was less prevalent among those with 1-9 teeth and the edentulous, and more prevalent among blacks, mixed-race, and indigenous, those who had never used dental service, with soft tissue alterations, who reported little pain or medium to intense pain, rated their own appearance and chewing as fair or bad or very bad, reported that their oral health limited their social interaction a little or considerably, and reported needing dental treatment $(P R=$ 1,47; 95\%CI: 1.20-1.79). Despite their precarious oral conditions, the majority gave their oral health a positive rating. Subjective conditions were more heavily associated with self-rated oral health than were objective conditions. The findings suggest unequal oral health conditions and allow orienting public policies aimed at oral health and quality of life.
\end{abstract}

Oral Health; Aged; Quality of Life
Andréa Maria Eleutério de Barros Lima Martins ${ }^{1,2}$ Sandhi Maria Barreto 3 Isabela Almeida Pordeus 4

\section{Introdução}

A auto-avaliação em saúde é a interpretação que uma pessoa faz de seu estado de saúde e experiências no contexto de sua vida diária. Esse julgamento se baseia, em geral, na informação e nos conhecimentos disponíveis de saúde e doença, mediados pela experiência prévia e pelo contexto social, cultural e histórico ${ }^{1}$. O conhecimento sobre a auto-avaliação da saúde da população contribui para orientar decisões políticas e sociais que tenham como meta a qualidade de vida e não meramente a saúde física. Conhecer os determinantes da auto-avaliação da saúde bucal é muito importante para entender o comportamento dos indivíduos e como os mesmos avaliam as suas necessidades. Na atenção odontológica individual, a investigação rotineira da auto-avaliação da saúde é importante para aumentar a adesão dos indivíduos a comportamentos saudáveis 2. Entre os idosos, isso é ainda mais relevante, pois mesmo nos países que têm programas dirigidos especificamente para esta faixa etária, a principal razão para este grupo não procurar o serviço odontológico é não perceber sua necessidade ${ }^{3}$.

A auto-avaliação da saúde bucal é uma variável multidimensional que reflete a experiência subjetiva dos indivíduos sobre o seu bem-estar funcional, social e psicológico 4,5. A complexidade dos fatores que influenciam esse julgamento tem sido evidenciada em diversos estudos 
$2,3,5,6,7,8,9,10,11$. Os fatores associados à auto-avaliação da saúde bucal podem ser relativos ao ambiente externo e ao indivíduo. $\mathrm{O}$ ambiente externo refere-se ao local de residência do indivíduo e ao sistema de atenção à saúde disponível, que pode ou não ofertar serviços odontológicos gratuitos que facilitem o acesso aos cuidados odontológicos. No nível individual, a auto-avaliação é influenciada por fatores direta ou indiretamente relacionados à saúde. Entre esses estão as características demográficas, como a idade, o sexo, a raça e fatores de predisposição, como escolaridade e acesso a informações sobre cuidados preventivos, que podem influenciar na predisposição para o uso de serviços odontológicos e conseqüentemente na auto-avaliação da saúde bucal. Também faz parte do nível individual a disponibilidade de recursos, incluindo renda pessoal e familiar, assim como a adesão a um plano de saúde que pode facilitar o acesso à atenção odontológica 8,9 .

O comportamento dos indivíduos pode ser influenciado ou influenciar a auto-avaliação da saúde bucal. Por exemplo, o uso de serviços pode ser conseqüência da auto-avaliação da saúde bucal ou mesmo esta auto-avaliação pode ser influenciada pelo uso de serviços odontológicos 12 . Além disso, o autocuidado que envolve a higiene bucal 5,6, o auto-exame da boca e a adesão a uma alimentação saudável têm sido associados à auto-avaliação da saúde bucal 8 .

A auto-avaliação dos indivíduos está associada a fatores objetivos e subjetivos. As condições objetivas mensuradas pela avaliação normativa feita pelo profissional estão correlacionadas à auto-avaliação da saúde bucal. Dentre os fatores já mencionados na literatura, destacam-se o número de dentes presentes, o número de dentes cariados e obturados presentes, o índice periodontal comunitário (CPI), o índice de perda de inserção (PIP), o edentulismo, o uso ou necessidade de próteses e a necessidade de tratamento $3,6,7,9$. No campo subjetivo, a saúde bucal autoavaliada está associada a outros julgamentos pessoais como a auto-avaliação da necessidade de tratamento odontológico ${ }^{9}$; a sensibilidade dolorosa nos dentes e gengivas 2; a auto-avaliação da aparência bucal, da mastigação, da fala e dos relacionamentos sociais em função das condições bucais 3,5,7,10. Finalmente, como seria de se esperar, a auto-avaliação da saúde bucal não está dissociada da saúde em geral, sofrendo a influência da presença de doenças sistêmicas e da saúde mental 2,13.

No Brasil, a saúde bucal da população idosa é precária 14,15. Não há dúvida de que a assistência pública odontológica precisa ser expandida e incrementada, especialmente entre os mais velhos.
Conhecer como os idosos auto-avaliam sua saúde bucal e os fatores que influenciam esta autoavaliação contribui para orientar mudanças nas políticas de saúde pública e assistencial na área, que necessitam incorporar tanto ações educativas de autocuidado como ações preventivas e reabilitadoras 3 .

O modelo teórico de Gift, Atchison \& Drury, proposto em 1998 9, adaptado pelos autores da presente investigação, prevê uma relação de retroalimentação entre cinco subgrupos de variáveis (ambiente externo, características individuais, comportamentos relacionados à saúde, condições objetivas e subjetivas de saúde) e a auto-avaliação da saúde geral e bucal. O modelo original não contemplava algumas variáveis, associadas à auto-avaliação da saúde bucal, identificadas em estudos posteriores. $\mathrm{O}$ ambiente externo (local de residência e sistema de atenção à saúde) e as características individuais (demográficas, de predisposição e de disponibilidade de recursos) influenciam os comportamentos relacionados à saúde (uso de serviços, autocuidado e hábitos). Os comportamentos, por sua vez, podem predizer ou serem conseqüência das condições objetivas (geral e bucal) e subjetivas (geral, bucal e qualidade de vida) relacionadas à saúde. As condições subjetivas influenciam e são influenciadas pelas condições objetivas. Considera-se ainda que as características individuais (de predisposição e de disponibilidade de recursos) e os comportamentos relacionados à saúde além de influenciar podem ser influenciados pelas condições objetivas e subjetivas de saúde (Figura 1). Portanto, o objetivo do presente estudo é identificar os fatores que estão associados à auto-avaliação negativa da saúde bucal dos idosos brasileiros utilizando como referencial teórico esse modelo.

\section{Métodos}

Foi utilizada a base de dados do inquérito de saúde bucal do Brasil (Projeto SB Brasil), realizado pelo Ministério da Saúde nos anos 2002/2003. Conforme proposta da Organização Mundial da Saúde (OMS), de 1997 16, além das condições sociodemográficas e uso de serviços odontológicos, o inquérito investigou, entre idosos, as condições (cárie, doença periodontal, necessidade de tratamento, uso e necessidade de prótese, alterações de tecidos moles) e a auto-avaliação da saúde bucal e da necessidade de tratamento odontológico 17.

O inquérito foi conduzido entre indivíduos residentes em 250 municípios, com representatividade garantida para o país e para as cinco 


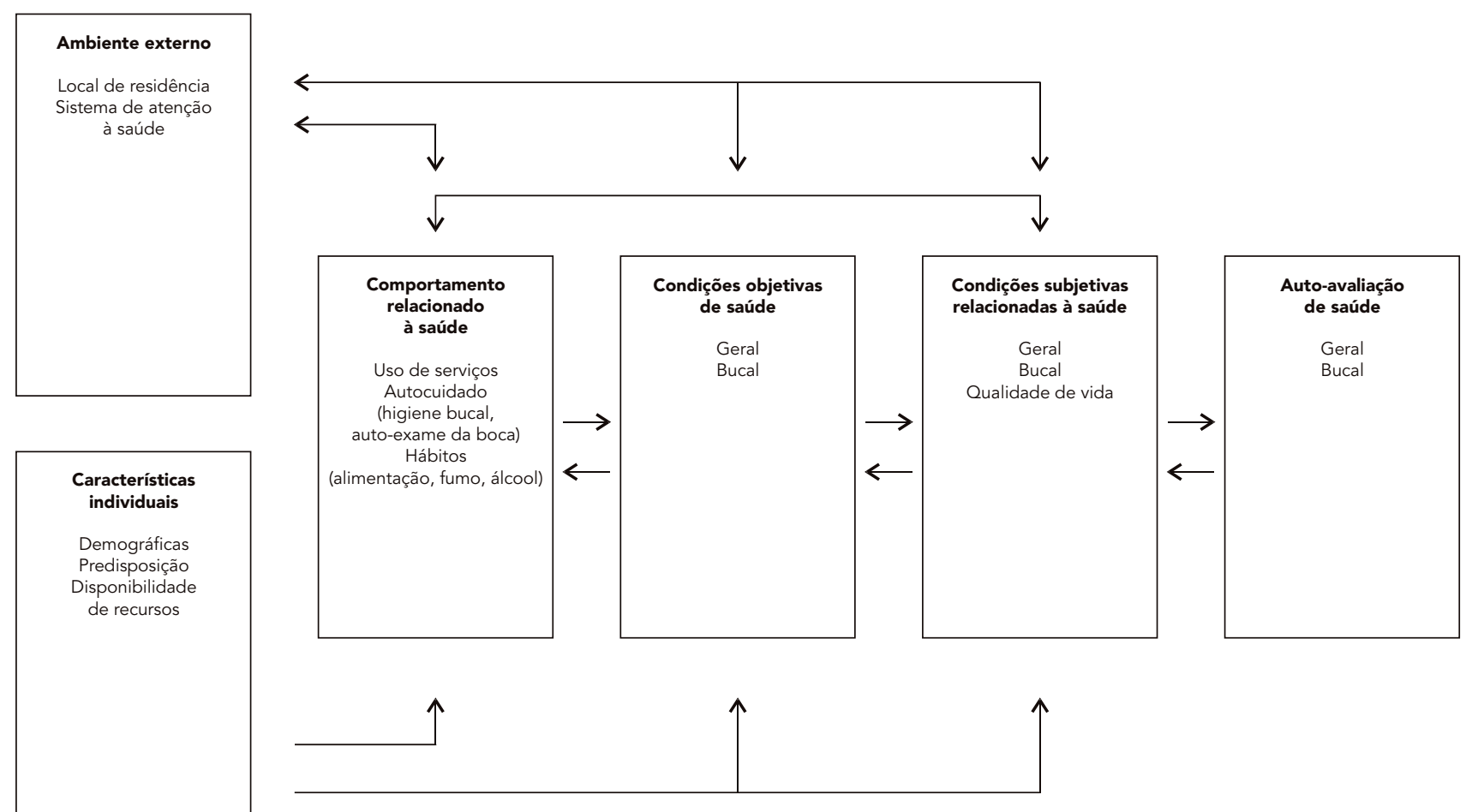

macrorregiões brasileiras, para duas idades índices (5 e 12 anos) e quatro estratos etários (18 a 36 meses, 15 a 19 anos, 35 a 44 anos e 65 a 74). A técnica de amostragem foi probabilística por conglomerados, sendo os indivíduos selecionados por meio de sorteio. Foram realizadas entrevistas e exames nos domicílios, sob iluminação natural, usando sonda periodontal (CPI), espelho bucal plano e espátulas de madeira para melhor visualização do campo examinado. Os exames foram conduzidos por dentistas treinados e calibrados (concordância kappa ou percentual). Os calibradores foram orientados a repetir o treinamento quando os resultados do teste kappa ou da concordância percentual não fossem satisfatórios 17 . Foi proposto que cerca de $5 \%$ dos exames fossem executados em duplicata com o objetivo de mensurar a concordância intra-examinador. O SB Brasil foi conduzido conforme os princípios éticos da Declaração de Helsinki contidos na Resolução no.196/96 do Conselho Nacional de Saúde e aprovado pela Comissão Nacional de Ética em Pesquisa (parecer no ${ }^{\circ} .581 / 2000$ ).
Maiores informações a respeito da metodologia adotada no projeto estão disponíveis em outras publicações 14,17,18.

A variável dependente estudada - auto-avaliação da condição de saúde bucal - foi obtida por meio da resposta à seguinte pergunta: "Como classificaria sua saúde bucal?” (péssima, ruim, regular, boa, ótima). As respostas foram agregadas em duas categorias: auto-avaliação ótima, boa ou regular - positiva - e auto-avaliação péssima ou ruim - negativa. Tal agregação seguiu aquela utilizada por Locker \& Gibson no Canadá 19 e por Borrel et al. 13 nos Estados Unidos. Essa agregação permite investigar o que influencia a auto-avaliação negativa da saúde, visto que esta espelha a condição mais crítica da saúde e da qualidade de vida dos indivíduos.

As variáveis independentes foram reunidas em cinco subgrupos definidos pelo modelo proposto: ambiente externo, características individuais, comportamento relacionado à saúde, condições objetivas de saúde, condições subjetivas relacionadas à saúde. 
O ambiente externo incluiu as variáveis "macrorregião do Brasil” (Sudeste, Sul, Centro-Oeste, Nordeste, Norte), "local de residência" (zona urbana, zona rural), "sistema de atenção à saúde utilizado" (plano/liberal/particular, público/filantrópico, nenhum), obtidas pela seqüência de duas perguntas: "Já foi ao dentista alguma vez na vida? Onde?". As características demográficas individuais investigadas foram: idade em anos (6569; 70-74); sexo (feminino, masculino); cor da pele autodeclarada (branco/amarelo, indígena/ pardo/negro). Os critérios de autoclassificação de cor/raça usado no SB Brasil são os mesmos do censo demográfico do Instituto Brasileiro de Geografia e Estatística (IBGE): pardos, pretos, brancos, amarelos (asiáticos) e indígenas. Entretanto, no presente trabalho adotou-se a palavra "negro" e não "preto" por ter sido esta a adotada no instrumento de coleta de dados do SB Brasil. As variáveis de predisposição foram a escolaridade em anos (5 ou mais, 1-4, 0) e o acesso a informações sobre como evitar problemas bucais (não, sim), obtidas a partir da pergunta: "Recebeu informações sobre como evitar problemas bucais?”. A disponibilidade de recursos foi avaliada pela renda domiciliar per capita em Reais categorizada em tercis ( $\mathrm{R} \$ 201,00$ ou mais; $\mathrm{R} \$ 100,00-\mathrm{R} \$ 200,00$; $\mathrm{R} \$$ 0,00-R\$ 99,00). O uso de serviços odontológicos (há dois anos ou menos, há mais de dois anos, nunca usou) foi a única variável referente ao comportamento investigada no inquérito, obtida pela seguinte pergunta: "Já foi ao dentista alguma vez na vida?".

As condições objetivas de saúde referem-se a indicadores padrões de doença: presença de alterações de tecidos moles (não, sim); número de dentes permanentes presentes (10 ou mais, 1-9, $0)$; número de dentes permanentes cariados presentes ( 0,1 a 3, 4 ou mais); necessidade de prótese (não necessita, necessita em uma arcada, necessita nas duas arcadas). A condição periodontal foi investigada entre idosos brasileiros, entretanto, não foi analisada na presente investigação por ser pertinente a apenas um estrato da população idosa (dentados) e pelo fato da presente análise não ter sido conduzida por estratos. As condições subjetivas relacionadas à saúde bucal incluíram auto-avaliação da dor nos dentes e gengivas nos últimos seis meses (nenhuma, pouca, média/ muita), obtida pela pergunta: "Quanto de dor seus dentes e gengivas causaram nos últimos seis meses?"; auto-avaliação da aparência dos dentes e gengivas (ótima/boa, regular, péssima/ruim) obtida pela pergunta: "Como classificaria a aparência dos seus dentes e gengivas?"; auto-avaliação da mastigação (ótima/boa, regular, péssima/ ruim) obtida pela pergunta: "Como classificaria sua mastigação?”; auto-avaliação da fala quanto aos dentes e gengivas (ótima/boa, regular, péssima/ruim) obtida pela pergunta: "Como classificaria sua fala devido a seus dentes e gengivas?”; auto-avaliação do relacionamento em função da saúde bucal (não afeta, afeta pouco, afeta mais ou menos /muito) obtida pela pergunta: "De que forma a sua saúde bucal afeta o seu relacionamento com outras pessoas?"; auto-avaliação da necessidade de tratamento (não, sim) obtida pela pergunta: "Considera que necessita de tratamento atualmente?".

Inicialmente foi feita a descrição das variáveis a fim de caracterizar a população. Foram realizados os testes de multicolinearidade entre as variáveis independentes. Considerou-se multicolinearidade elevada quando, simultaneamente, se observasse condition index maior que 30, que um componente contribuísse em $90 \%$ ou mais para a variância de duas ou mais variáveis, e se verificasse uma tolerância inferior a 0,1 (variance inflation factor - VIF) menor do que 10 20: a seguir, os idosos que auto-avaliaram sua saúde bucal como negativa foram comparados aos que a auto-avaliaram de forma positiva em relação a cada variável de interesse. Cada subgrupo definido pelo modelo proposto foi analisado separadamente. As magnitudes das associações entre a variável dependente e os fatores de interesse foram estimadas pelas razões de prevalências, com nível de significância de 0,05 e respectivos intervalos de 95\% de confiança (IC95\%). As razões de prevalências foram obtidas pela regressão de Poisson com estimação de variância robusta. A seguir, foi construído um modelo único, com base nos fatores retidos nos modelos intermediários. As variáveis que não estavam significativamente associadas e não contribuíam para o modelo foram eliminadas e o modelo final contém apenas os fatores que permaneceram associados em nível de $\mathrm{p} \leq 0,05$. Foi utilizado o programa Stata 9.0 (Stata Corp., College Station, Estados Unidos). A correção pelo efeito de desenho foi feita usandose o comando svy do Stata para a análise de dados oriundos de amostras complexas. Esse ajuste foi necessário porque a amostra do Projeto SB Brasil foi por conglomerados, e estimativas que não levam em consideração a organização por cluster da amostra tendem à superestimação e perda da precisão das estimativas 21 .

\section{Resultados}

O inquérito foi conduzido entre 108.921 indivíduos, $85 \%$ do total da amostra prevista (127.939 indivíduos). Entre os idosos foram entrevistados e examinados 5.349 indivíduos, sendo que pelo cálculo amostral deveriam ter participado do 
inquérito 3.800 idosos. Dos 5.014 idosos que responderam à pergunta sobre auto-avaliação da saúde bucal, $250(5 \%)$ a perceberam como péssima, 620 (12,4\%) como ruim, 1.465 (29,2\%) como regular, 2.452 (48,9\%) como boa e 227 (4,5\%) como ótima (Tabela 1). Com relação às condições objetivas de saúde, 2.931 (55\%), dentre os examinados, eram edentados e o índice CPOD médio foi igual a 28 . Do total de idosos, $10 \%$ apresentavam 20 ou mais dentes na boca. Um terço desses apresentava necessidade de prótese superior e mais da metade, de prótese inferior; 1.763 (33\%) usavam próteses. Quanto às alterações de tecidos moles, 863 (16\%) apresentavam algum tipo de alteração e 1.672 (31,3\%) idosos necessitavam de tratamento dentário.

Entre os 2.418 idosos dentados, o número médio de dentes permanentes presentes foi 12,1 (desvio-padrão - DP = 7,6), o número médio de dentes cariados por indivíduo foi 2,7 ( $\mathrm{DP}=3,8$ ); $1.983(82,4 \%)$ necessitavam de próteses. Somente 3 idosos (0,1\%) não necessitavam de tratamento dentário em pelo menos um dente remanescente, ou seja, praticamente todos os idosos brasileiros que tinham dentes remanescentes apresentavam a necessidade normativa de tratamento odontológico em pelo menos um dos dentes remanescentes. Além disso, entre os idosos dentados, uma proporção significativa [346 (14,3\%)] apresentava alterações de tecidos moles. Entre os 2.931 edentados, 1.074 (37\%) apresentavam necessidade de próteses, 517 (17,6\%) apresentavam alterações de tecidos moles e 1.418 (48,4\%) idosos tinham necessidade de prótese ou alterações de tecidos moles. Enfim, entre os dentados verificou-se a necessidade de tratamento odontológico em praticamente todos os idosos; já entre os edentados, pouco mais da metade $(51,6 \%)$ não apresentava alguma necessidade de tratamento odontológico.

A idade média dos idosos foi de 68,7 anos $(\mathrm{DP}=3,2)$. A maioria não possuía carro, grande parte era analfabeta $(1.874-35 \%)$ ou tinha menos de quatro anos de escolaridade (2.363 $44,2 \%$ ). A escolaridade média dos idosos foi de 2,7 anos ( $D P=3,1$ ), eles relataram uma baixa renda per capita, com média de $\mathrm{R} \$ 209,60$ (DP = R\$ $333,10)$ e $3.244(61 \%)$ idosos não receberam informações sobre como evitar problemas bucais.

$\mathrm{Na}$ análise univariada, verificou-se que entre os fatores referentes ao ambiente externo, às características individuais e ao comportamento, a maioria das variáveis esteve significativamente associada à auto-avaliação negativa da saúde bucal (Tabela 2). Quanto às variáveis referentes às condições objetivas e subjetivas, todas foram significativamente associadas à auto-avaliação negativa da saúde bucal (Tabela 3). Os testes de multicolinearidade demonstraram que todas as variáveis independentes propostas puderam ser analisadas em um modelo multivariado.

Na análise multivariada, utilizando regressão de Poisson, com estimação de variância robusta (Tabela 4), verificou-se que, quanto às características individuais, a prevalência da auto-avaliação negativa da saúde bucal foi maior entre pessoas classificadas como pardas ou negras e índias do que entre as brancas e amarelas. A auto-avaliação negativa da saúde bucal também foi mais freqüente entre indivíduos que nunca usaram serviços odontológicos. A condição objetiva de saúde que se manteve diretamente associada à auto-avaliação negativa foi a presença de alterações de tecidos moles, e inversamente associada à auto-avaliação foi o menor número de dentes e o edentulismo. As condições subjetivas relacionadas à saúde que se mantiveram positivamente associadas à auto-avaliação negativa da saúde bucal entre idosos foram: relato de dor nos dentes e gengivas nos últimos seis meses; auto-avaliação da aparência e mastigação como regular, ruim ou péssima em função dos dentes ou gengivas; perceber o relacionamento social afetado pelas condições de saúde bucal; e auto-avaliar a necessidade de tratamento odontológico. As variáveis independentes de auto-avaliação da dor, da aparência, da mastigação e do relacionamento apresentaram claro gradiente dose resposta.

Finalmente, foram testadas as interações entre condições clínicas (número de dentes presentes na boca e alterações de tecido mole) e condições sócio-demográficas selecionadas (sexo, cor da pele e escolaridade), mas não foram encontradas evidências de heterogeneidade.

\section{Discussão}

Como em outros estudos internacionais e nacionais $2,3,6,7,9,10,11,22,23,24,25,26,27,28$, o presente trabalho mostrou um predomínio da auto-avaliação positiva da saúde bucal. Tal avaliação parece antagônica tendo em vista as precárias condições objetivas de saúde bucal dos idosos. Além do edentulismo que atinge metade da população idosa, a análise dos dentados mostrou que a maioria apresentava poucos dentes remanescentes e alguma necessidade de tratamento. Apenas $10 \%$ dos idosos atingiram a meta da OMS para o ano 2000 29, que preconizava que no mínimo $50 \%$ deles apresentassem 20 ou mais dentes na boca.

Vale ressaltar, no entanto, que os idosos analisados no presente trabalho viveram uma época em que o edentulismo e condições precárias da dentição, assim como o adoecimento, parecem ter sido considerados parte do processo "nor- 
Tabela 1

Caracterização dos idosos, segundo auto-avaliação da saúde bucal, ambiente externo, características individuais, comportamento relacionando à saúde, condições objetivas de saúde e condições subjetivas relacionadas à saúde. Projetos SB Brasil, 2002/2003.

n

$\begin{array}{cc}4.144 & 82,6 \\ 870 & 17,4\end{array}$

Ambiente externo

Macrorregião do Brasi

Sudeste

Sul

Centro-Oeste

Nordeste

Norte

1.000

1.326

672

1.317

699

4.370

642

Zona urbana

Zona rural

Sistema da atenção à saúde utilizado

Plano/Liberal/Particular

2.482

2.106

Público/Filantrópico

Nenhum

198

Características individuais

Demográficas

Idade (anos)

65-69

70-74

3.015

Sexo

Feminino

Masculino

1.999

3.049

1.965

2.615

Branco/Amarelo

Pardo/Negro/Índio

Predisposição

Escolaridade (anos)

5 ou mais

988

2.277

1.749

0

Informações sobre como evitar problemas bucais

Sim

2.024

2.987

1.489

1.966

1.527

$0,00-99,00$

Comportamento

Uso de serviços odontológicos

Há 2 anos ou menos

Há mais de 2 anos

1.470

3.331

Nunca usou
60,1

$\%$

17,

20,0

26,4

13,4

26,3

13,9

87,2

12,8

51,9

44,0

4,1

399

60,8

39,2

52,3

47,7

19,7

45,4

34,9

40,4

59,6

29,9

39,5

30,6

29,4

66,6

4,0

(continua) 
Condições objetivas

Alterações de tecidos moles Não

Sim

$\begin{array}{cc}4.164 & 83,6 \\ 814 & 16,4 \\ & \\ 1.208 & 24,1 \\ 1.079 & 21,5 \\ 2.727 & 54,4\end{array}$

Número de dentes permanentes cariados presentes 0

$1-3$

3.542

70,6

667

13,3

4 ou mais

80516,1

Necessidade de prótese

Não necessita

Necessita em uma arcada

Necessita nas duas arcadas

1.131

26,2

1.521

Condições subjetivas

Auto-avaliação da dor

Nenhuma

Pouca

Média/Muita

Auto-avaliação da aparência

Ótima/Boa

Regular

$1.407 \quad 29,6$

Péssima/Ruim

$972 \quad 20,5$

Auto-avaliação da mastigação

Ótima/Boa

$2.519 \quad 50,6$

Regular

$1.230 \quad 24,7$

Péssima/Ruim

$1.229 \quad 24,7$

Auto-avaliação da fala

Ótima/Boa

$3.060 \quad 62,9$

$1.102 \quad 22,6$

Regular

Péssima/Ruim

708

Auto-avaliação do relacionamento

Não afeta

Afeta pouco

Afeta mais ou menos/muito

Auto-avaliação da necessidade de tratamento

Não

mal" de envelhecimento. Essas condições precárias parecem refletir o modelo de atenção à saúde adotado no país, com acesso muito restrito e desigual, atenção básica precária e assistência mutiladora 30. É possível, portanto, que a autoavaliação positiva da saúde bucal, em condições tão precárias, reflita, em parte, uma atitude de resignação culturalmente difundida 3 .

A aparente inconsistência entre predomínio de condição objetiva ruim e de auto-avaliação positiva da saúde bucal também foi relatada por diversos autores $3,6,9,10$, e provavelmente reflete o fato de as condições objetivas serem fracamente associadas à auto-avaliação 6,7. Uma das explicações para essa fraca associação deve-se ao fato de muitas doenças detectadas pelo profissional ao exame serem assintomáticas e desconhecidas pelos indivíduos 6,31. Ou seja, os idosos tendem a se considerar doentes mediante manifestações agudas de doenças bucais e não frente a processos crônicos e irreversíveis destas doenças que levam a perdas dentais 6 . Outra explicação para 
Tabela 2

Análise univariada da auto-avaliação da saúde bucal segundo as variáveis referentes ao ambiente externo, às características individuais e ao comportamento, entre idosos. Projeto SB Brasil, 2002/2003.

\begin{tabular}{|c|c|c|c|c|c|c|c|}
\hline & \multicolumn{7}{|c|}{ Auto-avaliação } \\
\hline & \multicolumn{2}{|c|}{ Positiva } & \multicolumn{2}{|c|}{ Negativa } & \multirow[t]{2}{*}{ RP } & \multirow[t]{2}{*}{ IC95\% } & \multirow{2}{*}{$\begin{array}{c}\text { Valor de } \\
\qquad p\end{array}$} \\
\hline & n & $\%$ & $\mathrm{n}$ & $\%$ & & & \\
\hline \multicolumn{8}{|l|}{ Ambiente externo } \\
\hline \multicolumn{8}{|l|}{ Macrorregião do Brasil } \\
\hline Sudeste & 870 & 21,0 & 130 & 14,9 & 1,00 & & \\
\hline Sul & 1.172 & 28,3 & 154 & 17,7 & 0,89 & $0,65-1,21$ & 0,46 \\
\hline Centro-Oeste & 560 & 13,5 & 112 & 12,9 & 1,28 & $0,93-1,76$ & 0,12 \\
\hline Nordeste & 1.014 & 24,5 & 303 & 34,8 & 1,76 & $1,32-2,36$ & $<0,01$ \\
\hline Norte & 528 & 12,7 & 171 & 19,7 & 1,88 & $1,37-2,56$ & $<0,01$ \\
\hline \multicolumn{8}{|l|}{ Local de residência * } \\
\hline Zona urbana & 3.613 & 87,2 & 757 & 87,2 & 1,00 & & \\
\hline Zona rural & 531 & 12,8 & 111 & 12,8 & 0,99 & $0,80-1,23$ & 0,98 \\
\hline \multicolumn{8}{|c|}{ Sistema da atenção à saúde utilizado * } \\
\hline Plano/Liberal/Particular & 2.162 & 54,4 & 320 & 39,6 & 1,00 & & \\
\hline Público/Filantrópico & 1.704 & 42,8 & 402 & 49,7 & 1,48 & $1,28-1,70$ & $<0,01$ \\
\hline Nenhum & 111 & 2,8 & 87 & 10,8 & 3,40 & $2,75-4,22$ & $<0,01$ \\
\hline \multicolumn{8}{|l|}{ Características individuais } \\
\hline \multicolumn{8}{|l|}{ Demográficas } \\
\hline \multicolumn{8}{|l|}{ Idade (anos) } \\
\hline $65-69$ & 2.456 & 59,3 & 559 & 64,3 & 1,00 & & \\
\hline $70-74$ & 1.688 & 40,7 & 311 & 35,7 & 0,83 & $0,73-0,95$ & $<0,01$ \\
\hline \multicolumn{8}{|l|}{ Sexo } \\
\hline Feminino & 2.560 & 61,8 & 489 & 56,2 & 1,00 & & \\
\hline Masculino & 1.584 & 38,2 & 381 & 43,8 & 1,20 & $1,06-1,36$ & $<0,01$ \\
\hline \multicolumn{8}{|l|}{ Cor da pele autodeclarada * } \\
\hline Branco/Amarelo & 2.276 & 55,1 & 339 & 39,0 & 1,00 & & \\
\hline Pardo/Negro/Índio & 1.854 & 44,9 & 530 & 61,0 & 1,71 & $1,48-1,98$ & $<0,01$ \\
\hline \multicolumn{8}{|l|}{ Predisposição } \\
\hline \multicolumn{8}{|l|}{ Escolaridade (anos) } \\
\hline 5 ou mais & 838 & 20,2 & 150 & 17,2 & 1,00 & & \\
\hline $1-4$ & 1.930 & 46,6 & 347 & 39,9 & 1,08 & $0,88-1,33$ & 0,42 \\
\hline 0 & 1.376 & 33,2 & 373 & 42,9 & 1,45 & $1,22-1,72$ & $<0,01$ \\
\hline \multicolumn{8}{|c|}{ Informações sobre como evitar problemas bucais * } \\
\hline Sim & 1.742 & 42,1 & 282 & 32,4 & 1,00 & & \\
\hline Não & 2.399 & 57,9 & 588 & 67,6 & 1,41 & $1,20-1,65$ & $<0,01$ \\
\hline \multicolumn{8}{|l|}{ Disponibilidade de recursos } \\
\hline \multicolumn{8}{|l|}{ Renda (Reais)* } \\
\hline 201,00 ou mais & 1.314 & 31,9 & 175 & 20,3 & 1,00 & & \\
\hline $100,00-200,00$ & 1.637 & 39,7 & 329 & 38,2 & 1,42 & $1,18-1,71$ & $<0,01$ \\
\hline $0,00-99,00$ & 1.169 & 28,4 & 358 & 41,5 & 1,99 & $1,65-2,40$ & $<0,01$ \\
\hline \multicolumn{8}{|l|}{ Comportamento } \\
\hline \multicolumn{8}{|c|}{ Uso de serviços odontológicos * } \\
\hline Há 2 anos ou menos & 1.227 & 29,7 & 243 & 28,0 & 1,00 & & \\
\hline Há mais de 2 anos & 2.793 & 67,6 & 538 & 62,0 & 0,97 & $0,80-1,18$ & 0,81 \\
\hline Nunca usou & 111 & 2,7 & 87 & 10,0 & 2,65 & $2,07-3,41$ & $<0,01$ \\
\hline
\end{tabular}

RP: razão de prevalência; IC95\%: intervalo de 95\% de confiança.

* Os totais variam devido às perdas de informação. 
Análise univariada da auto-avaliação da saúde bucal segundo as variáveis referentes às condições objetivas e subjetivas de saúde, entre idosos. Projeto SB Brasil, 2002/2003.

\begin{tabular}{|c|c|c|c|c|c|c|c|}
\hline & \multicolumn{7}{|c|}{ Auto-avaliação } \\
\hline & \multicolumn{2}{|c|}{ Positiva } & \multicolumn{2}{|c|}{ Negativa } & \multirow[t]{2}{*}{ RP } & \multirow[t]{2}{*}{ IC95\% } & \multirow{2}{*}{$\begin{array}{c}\text { Valor de } \\
\text { p }\end{array}$} \\
\hline & $\mathrm{n}$ & $\%$ & $\mathrm{n}$ & $\%$ & & & \\
\hline \multicolumn{8}{|l|}{ Condições objetivas } \\
\hline \multicolumn{8}{|l|}{ Alterações de tecidos moles * } \\
\hline Sudeste & 3.485 & 84,7 & 679 & 78,7 & 1,00 & & \\
\hline Sul & 630 & 15,3 & 184 & 21,3 & 1,38 & $1,20-1,65$ & $<0,01$ \\
\hline \multicolumn{8}{|c|}{ Número de dentes permanentes presentes } \\
\hline 10 ou mais & 903 & 21,8 & 305 & 35,1 & 1,00 & & \\
\hline $1-9$ & 823 & 19,9 & 256 & 29,4 & 0,93 & $0,80-1,10$ & 0,43 \\
\hline 0 & 2.418 & 58,3 & 309 & 35,5 & 0,44 & $0,37-0,53$ & $<0,01$ \\
\hline \multicolumn{8}{|c|}{ Número de dentes permanentes cariados presentes } \\
\hline 0 & 3.104 & 74,9 & 438 & 50,3 & 1,00 & & \\
\hline $1-3$ & 524 & 12,6 & 143 & 16,4 & 1,73 & $1,42-2,11$ & $<0,01$ \\
\hline 4 ou mais & 516 & 12,5 & 289 & 33,2 & 2,90 & $2,47-3,39$ & $<0,01$ \\
\hline \multicolumn{8}{|l|}{ Necessidade de prótese * } \\
\hline Não necessita & 1.961 & 47,5 & 202 & 23,4 & 1,00 & & \\
\hline Necessita em uma arcada & 1.113 & 26,9 & 198 & 22,9 & 1,61 & $1,28-2,03$ & $<0,01$ \\
\hline Necessita nas duas arcadas & 1.057 & 25,6 & 464 & 53,7 & 3,26 & $2,66-4,00$ & $<0,01$ \\
\hline \multicolumn{8}{|l|}{ Condições subjetivas } \\
\hline \multicolumn{8}{|l|}{ Auto-avaliação da dor } \\
\hline Nenhuma & 3.359 & 81,1 & 499 & 57,5 & 1,00 & & \\
\hline Pouca & 513 & 12,4 & 169 & 19,5 & 1,91 & $1,53-2,39$ & $<0,01$ \\
\hline Média/Muita & 270 & 6,5 & 200 & 23,0 & 3,28 & $2,72-3,96$ & $<0,01$ \\
\hline \multicolumn{8}{|l|}{ Auto-avaliação da aparência * } \\
\hline Ótima/Boa & 2.271 & 57,9 & 100 & 12,1 & 1,00 & & \\
\hline Regular & 1.270 & 32,4 & 137 & 16,6 & 2,30 & $1,78-2,98$ & $<0,01$ \\
\hline Péssima/Ruim & 384 & 9,8 & 588 & 71,3 & 14,34 & $11,71-17,55$ & $<0,01$ \\
\hline \multicolumn{8}{|l|}{ Auto-avaliação da mastigação * } \\
\hline Ótima/Boa & 2.386 & 57,9 & 133 & 15,6 & 1,00 & & \\
\hline Regular & 1.077 & 26,2 & 153 & 17,8 & 2,35 & $1,88-2,94$ & $<0,01$ \\
\hline Péssima/Ruim & 655 & 15,9 & 574 & 66,7 & 8,84 & $7,25-10,79$ & $<0,01$ \\
\hline \multicolumn{8}{|l|}{ Auto-avaliação da fala * } \\
\hline Ótima/Boa & 2.779 & 68,9 & 281 & 33,7 & 1,00 & & \\
\hline Regular & 912 & 22,6 & 190 & 22,8 & 1,87 & $1,53-2,29$ & $<0,01$ \\
\hline Péssima/Ruim & 344 & 8,5 & 364 & 43,6 & 5,59 & $4,66-6,71$ & $<0,01$ \\
\hline \multicolumn{8}{|c|}{ Auto-avaliação do relacionamento * } \\
\hline Não afeta & 2.910 & 77,5 & 342 & 44,4 & 1,00 & & \\
\hline Afeta pouco & 463 & 12,3 & 197 & 25,6 & 2,83 & $2,35-3,42$ & $<0,01$ \\
\hline Afeta mais ou menos/muito & 383 & 10,2 & 231 & 30,0 & 3,57 & $2,94-4,35$ & $<0,01$ \\
\hline \multicolumn{8}{|c|}{ Auto-avaliação da necessidade de tratamento * } \\
\hline Não & 2.114 & 51,1 & 169 & 19,4 & 1,00 & & \\
\hline Sim & 2.022 & 48,9 & 700 & 80,6 & 3,47 & $2,77-4,34$ & $<0,01$ \\
\hline
\end{tabular}

RP: razão de prevalência; IC95\%: intervalo de 95\% de confiança.

* Os totais variam devido às perdas de informação. 
Análise multivariada dos fatores associados à auto-avaliação da saúde bucal, entre idosos. Projeto SB Brasil, $2002 / 2003$.

\begin{tabular}{|c|c|c|c|c|c|c|}
\hline & \multicolumn{6}{|c|}{ Auto-avaliação } \\
\hline & \multirow{2}{*}{$\begin{array}{c}\text { Positiva } \\
\text { (\%) }\end{array}$} & \multirow{2}{*}{$\begin{array}{c}\text { Negativa } \\
\text { (\%) }\end{array}$} & \multicolumn{2}{|c|}{ Modelo bruto } & \multicolumn{2}{|c|}{ Modelo ajustado } \\
\hline & & & RP & IC95\% & RP & IC95\% \\
\hline \multicolumn{7}{|l|}{ Características individuais } \\
\hline \multicolumn{7}{|l|}{ Demográficas } \\
\hline \multicolumn{7}{|l|}{ Cor da pele autodeclarada } \\
\hline Branco/Amarelo & 55,1 & 39,0 & 1,00 & & 1,00 & \\
\hline Pardo/Negro/Índio & 44,9 & 61,0 & 1,71 & $1,48-1,98$ & 1,19 & $1,05-1,34$ \\
\hline \multicolumn{7}{|l|}{ Comportamento } \\
\hline \multicolumn{7}{|l|}{ Uso de serviços odontológicos } \\
\hline Há 2 anos ou menos & 29,7 & 28 & 1,00 & & 1,00 & \\
\hline Há mais de 2 anos & 67,6 & 62 & 0,97 & $0,80-1,18$ & 1,12 & $0,96-1,32$ \\
\hline Nunca usou & 2,7 & 10 & 2,65 & $2,07-3,41$ & 1,35 & $1,08-1,69$ \\
\hline \multicolumn{7}{|l|}{ Condições objetivas } \\
\hline \multicolumn{7}{|l|}{ Alterações de tecidos moles } \\
\hline Não & 84,7 & 78,7 & 1,00 & & 1,00 & \\
\hline Sim & 15,3 & 21,3 & 1,38 & $1,20-1,65$ & 1,18 & $1,02-1,37$ \\
\hline \multicolumn{7}{|c|}{ Número de dentes permanentes presentes } \\
\hline 10 ou mais & 21,8 & 35,1 & 1,00 & & 1,00 & \\
\hline $1-9$ & 19,9 & 29,4 & 0,93 & $0,80-1,10$ & 0,82 & $0,72-0,94$ \\
\hline 0 & 58,3 & 35,5 & 0,44 & $0,37-0,53$ & 0,71 & $0,61-0,83$ \\
\hline \multicolumn{7}{|l|}{ Condições subjetivas } \\
\hline \multicolumn{7}{|l|}{ Auto-avaliação da dor } \\
\hline Nenhuma & 81,1 & 57,5 & 1,00 & & 1,00 & \\
\hline Pouca & 12,4 & 19,5 & 1,91 & $1,53-2,39$ & 1,27 & $1,05-1,55$ \\
\hline Média/Muita & 6,5 & 23,0 & 3,28 & $2,72-3,96$ & 1,32 & $1,13-1,55$ \\
\hline \multicolumn{7}{|l|}{ Auto-avaliação da aparência } \\
\hline Ótima/Boa & 57,9 & 12,1 & 1,00 & & 1,00 & \\
\hline Regular & 32,4 & 16,6 & 2,30 & $1,78-2,98$ & 1,51 & $1,15-1,97$ \\
\hline Péssima/Ruim & 9,8 & 71,3 & 14,34 & $11,7-17,5$ & 5,32 & $4,14-6,84$ \\
\hline \multicolumn{7}{|l|}{ Auto-avaliação da mastigação } \\
\hline Ótima/Boa & 57,9 & 15,6 & 1,00 & & 1,00 & \\
\hline Regular & 26,2 & 17,8 & 2,35 & $1,88-2,94$ & 1,49 & $1,19-1,88$ \\
\hline Péssima/Ruim & 15,9 & 66.7 & 8,84 & $7,25-10,8$ & 2,83 & $2,25-3,57$ \\
\hline \multicolumn{7}{|l|}{ Auto-avaliação do relacionamento } \\
\hline Não afeta & 77,5 & 44,4 & 1,00 & & 1,00 & \\
\hline Afeta pouco & 12,3 & 25,6 & 2,83 & $2,35-3,42$ & 1,20 & $1,02-1,42$ \\
\hline Afeta mais ou menos/muito & 10,2 & 30,0 & 3,57 & $2,94-4,35$ & 1,26 & $1,08-1,47$ \\
\hline \multicolumn{7}{|c|}{ Auto-avaliação da necessidade de tratamento } \\
\hline Não & 51,1 & 19,4 & 1,00 & & 1,00 & \\
\hline Sim & 48,9 & 80,6 & 3,47 & $2,77-4,34$ & 1,47 & $1,20-1,79$ \\
\hline
\end{tabular}

RP: razão de prevalência; IC95\%: intervalo de 95\% de confiança.

essas associações fracas ou inconsistentes seria a suposição de que indicadores objetivos medem doença, enquanto os subjetivos avaliam experiências humanas e saúde 32 . As diferenças entre a auto-avaliação e as condições objetivas de saúde bucal provavelmente são a principal razão para os indivíduos não procurarem os serviços odontológicos quando estes estão disponíveis.
Quando essa disparidade entre condições objetivas e subjetivas de saúde não é considerada, a organização e a oferta de serviços odontológicos têm pequeno efeito nos padrões de uso destes serviços 6, ou mesmo nas mudanças de comportamento para um estilo de vida mais saudável. No presente estudo, as associações entre condições objetivas e a auto-avaliação da saúde bucal tam- 
bém foram inconsistentes. Verificaram-se ainda associações com alterações de tecidos moles e número de dentes permanentes presentes.

Os fatores referentes ao ambiente externo não foram significativos no modelo final. Entre as variáveis referentes às características individuais, somente a raça/cor autodeclarada esteve associada à auto-avaliação negativa da saúde bucal entre idosos. A associação entre condições demográficas e auto-avaliação da saúde bucal foi investigada por outros autores. Os resultados quanto à idade são inconsistentes, alguns estudos evidenciaram que a auto-avaliação negativa da saúde bucal aumenta com a idade 8,9; em outro estudo, entretanto, verificou-se que os mais velhos relatavam uma auto-avaliação mais positiva da saúde bucal 6; já em outras investigações, a idade não esteve associada à auto-avaliação 3,7 , concordando com o observado na presente investigação. A maioria dos estudos também não constatou associação entre sexo e auto-avaliação $3,8,9$, embora uma pesquisa tenha relatado pior auto-avaliação da saúde bucal entre mulheres 6 . Como na presente investigação, alguns estudos mostraram que indivíduos brancos auto-avaliaram sua saúde bucal de forma mais positiva do que os não-brancos 7,8. Esse achado sugere a presença de iniqüidades e concorre para o reconhecimento das diferenças entre os grupos étnicos que compõem a sociedade brasileira. Os afrobrasileiros, por razões de violação continuada de seus direitos humanos fundamentais, merecem tratamento diferenciado do poder público, de modo a eliminar ou reduzir a níveis não significativos as iniqüidades existentes 33 .

As variáveis de predisposição e de disponibilidade de recursos pouco contribuíram para a identificação dos fatores associados à auto-avaliação. Outras investigações demonstraram que quanto maior a escolaridade do indivíduo 7,8,9,22 e o acesso a informações sobre como evitar problemas bucais, mais positiva tende a ser a sua auto-avaliação da saúde bucal 8,9. A literatura é controversa quanto à renda. Em dois estudos foi verificada uma auto-avaliação mais positiva entre aqueles com maior renda 8,34 , já em outra investigação não foi constatada nenhuma associação entre renda e auto-avaliação da saúde bucal ${ }^{9}$. Na presente investigação, essa associação não foi verificada provavelmente devido à maior homogeneidade da população, já que a maioria relatou baixa renda.

A variável referente ao comportamento, "uso de serviços odontológicos", esteve significativamente associada à auto-avaliação negativa da saúde bucal. Outra investigação também constatou que os indivíduos que usaram os serviços odontológicos há mais de um ano relataram uma auto-avaliação da saúde bucal mais negativa 8 .

Dentre as condições objetivas de saúde, dois indicadores (alterações de tecidos moles e número de dentes permanentes presentes) estiveram associados à auto-avaliação negativa da saúde bucal. Essas condições parecem ter limitada relevância na auto-avaliação da saúde bucal 6 . O número de dentes permanentes cariados presentes foi significativamente associado à auto-avaliação apenas na análise univariada. Diversos estudos constataram que o maior número de dentes cariados presentes na boca dos indivíduos foi associado a uma auto-avaliação negativa da saúde bucal 6,9,25,34, mas, em geral, o percentual de idosos com maior número de dentes é bem superior 6 ao observado no Brasil.

A variável "alterações de tecidos moles" foi utilizada no SB Brasil com o intuito de realizar um "rastreamento", podendo referir-se a diversas manifestações, desde uma pequena lesão a ocorrências mais graves. Porém, aqueles que apresentaram alterações nos tecidos moles também apresentaram maior freqüência de auto-avaliação negativa da saúde bucal, mas, infelizmente, não identificamos nenhuma publicação que tenha investigado esta questão. É possível que a associação encontrada no presente estudo esteja relacionada a sintomas freqüentes em portadores de lesões como a halitose 35 , alteração de paladar 36 e dor. Mas, essa possibilidade necessita ser avaliada em estudos futuros que investiguem esses sintomas.

O número de dentes permanentes foi significativo e negativamente associado à auto-avaliação. Verificou-se um gradiente inverso, com menor prevalência de auto-avaliação negativa entre aqueles que tinham de 1 a 9 ou nenhum dente, quando comparados àqueles que tinham dez ou mais dentes. Apesar da aparente incoerência desses resultados, os idosos parecem avaliar positivamente sua saúde bucal estando livres de dentes cariados e possivelmente de doenças, do que com a manutenção de poucos dentes em precárias condições, em número insuficiente e sem acesso a próteses para assegurar uma mastigação eficiente e confortável. Tal resultado difere de investigações prévias que demonstraram que o maior número de dentes permanentes presentes esteve associado a uma auto-avaliação da saúde bucal mais positiva 6,7,8,9,25. Entretanto, o CPOD médio dos participantes de uma dessas investigações foi menor do que o encontrado entre idosos brasileiros $(20,4) 6$. Além disso, nessas investigações a maioria dos dentes remanescentes dos participantes estava obturado 6,7 e não extraído como o constatado entre idosos brasileiros na presente investigação. Sabe-se também que os 
idosos edêntulos normalmente acreditam que não necessitam de tratamento, o que foi evidenciado pela menor prevalência de uso de serviços odontológicos, no último ano, entre os idosos que apresentavam um maior número de dentes quando comparados àqueles com um menor número ou eram edentados 15 , o que poderia ser outra explicação para a menor prevalência de auto-avaliação negativa da saúde bucal entre idosos brasileiros edêntulos.

As condições subjetivas foram as que estiveram mais associadas à auto-avaliação da saúde bucal, indicando que as questões relativas ao impacto que a condição bucal exerce sobre a qualidade de vida dos indivíduos são mais determinantes da auto-avaliação do que as questões objetivas da condição de saúde bucal. A autoavaliação da aparência dos dentes e gengivas foi a variável que esteve mais fortemente associada, o que foi verificado por outros autores 7,25. A auto-avaliação da mastigação, da necessidade de tratamento, do relacionamento social em função da condição bucal e o relato de sensibilidade dolorosa nos dentes ou gengivas nos últimos seis meses também estiveram associados. Associações entre auto-avaliação da saúde bucal de uma forma global e auto-avaliações específicas de limitações funcionais ou psicológicas foram observadas em outra investigação 37 . A associação entre sensibilidade dolorosa e autoavaliação negativa da saúde bucal constatada na presente investigação também foi verificada em outros estudos 8,22 .

A utilização de serviços odontológicos (preventivo, curativo e reabilitador) traduz a autoavaliação da saúde, das necessidades, dos desejos e das demandas dos indivíduos 12, sendo mediada pela oferta, acesso e qualidade dos serviços em uma dada sociedade. Assim como os resultados apresentados, outras investigações também mostraram 9,11,25,34,38 que a auto-avaliação positiva da saúde bucal foi maior entre aqueles que relataram não necessitar de tratamento. Mesmo considerando que o tratamento odontológico pode também ser deletério para a dentição ${ }^{39}$, é difícil crer que a condição dentária dos idosos seja explicada pela exposição a tratamentos inadequados, visto que a oferta de serviços odontológicos era pouco freqüente quando estes idosos eram crianças e jovens, fase essencial para uma dentição sadia, e que alguns idosos jamais usaram estes serviços 15,40. Vale lembrar também que os idosos atuais não foram expostos à fluoretação da água e da pasta dental, o que com certeza concorreu para a precariedade da saúde bucal dos mesmos no presente 3,30 .

O Projeto SB Brasil teve um caráter exploratório no que diz respeito a informações subjetivas e a algumas informações objetivas da saúde bucal dos idosos. Entretanto, o uso dos dados desse inquérito para investigar associações tem permitido aumentar o conhecimento sobre a saúde desse grupo populacional e precisa ser estimulado, inclusive para subsidiar melhorias em um próximo inquérito desta natureza no país. A metodologia amostral, a participação dos idosos e os procedimentos adotados pelo Projeto SB Brasil visaram garantir as validades interna e externa dos resultados, muito embora o cálculo do tamanho amostral tenha usado como parâmetro o CPOD médio de um inquérito anterior, não adequado para estimar a amostra de um inquérito que investigou outras condições objetivas, assim como condições subjetivas. Além disso, nem todas as variáveis potencialmente relacionadas à auto-avaliação da saúde, contempladas no modelo proposto, foram investigadas no Projeto SB Brasil, o que impediu testar plenamente o modelo na presente análise. O processo que relaciona a auto-avaliação da saúde bucal e as variáveis investigadas é dinâmico, conforme indica a ação de retroalimentação do modelo adotado. O SB Brasil foi um estudo transversal, por isto não permite estabelecer uma relação temporal entre as associações encontradas, o que limita, até certo ponto, a interpretação dos resultados apresentados. Finalmente, é importante salientar que a auto-avaliação da saúde bucal do indivíduo muda ao longo da vida e que pode mudar até mesmo ao longo de um dia ou de uma semana em função do seu estado físico e psicológico e de condições contextuais. Portanto, captar a subjetividade do bem-estar e do adoecer, tentar quantificar o que é tão subjetivo é sempre limitado, pois envolve também valores e sentimentos nem sempre expressos.

Apesar das precárias condições dentárias dos idosos brasileiros, predominou a auto-avaliação positiva da saúde bucal. Condições subjetivas, relacionadas ao impacto na qualidade de vida, estiveram mais fortemente associadas à auto-avaliação negativa da saúde bucal do que condições objetivas de saúde. Sendo assim, as medidas objetivas são insuficientes para avaliar a saúde bucal. A maior prevalência de auto-avaliação negativa da saúde bucal, entre os que nunca usaram os serviços odontológicos, sugere que $o$ atendimento de forma regular possivelmente manteria o idoso informado e com maior conhecimento para avaliar a necessidade de tratamento, o que poderia influenciar o seu comportamento e qualidade de vida. Nesses serviços, o tratamento das alterações de tecidos moles, o tratamento ou remoção de dentes em precárias condições, o tratamento periodontal, a reabilitação protética e considerações sobre a 
avaliação dos idosos a respeito das suas condições bucais de saúde poderiam contribuir para melhorar a qualidade de vida e de saúde desse estrato da população.

O melhor entendimento das interações entre a auto-avaliação da saúde bucal e o ambiente externo, as características individuais, o comportamento relacionado à saúde, as condições objetivas e subjetivas de saúde serão possíveis mediante o fortalecimento de bases conceituais e o incremento de investigações sobre estas questões. Análises longitudinais com a inserção de outras variáveis que possam estar associadas à auto-avaliação da saúde bucal poderiam incrementar a compreensão do que interfere nesta auto-avaliação, no comportamento dos idosos e, conseqüentemente, na sua qualidade de vida e saúde.

\section{Resumo}

Propôs-se investigar fatores associados à auto-avaliação negativa da saúde bucal. Idosos, participantes do inquérito de saúde bucal do Ministério da Saúde, 2002-2003, que auto-avaliaram sua saúde bucal como ruim/péssima foram comparados aos que a auto-avaliaram como ótima/boa/regular usando-se razões de prevalências (RP) com base na regressão de Poisson. A minoria (870 - 17\%) auto-avaliou sua saúde bucal negativamente. A auto-avaliação negativa foi menor entre aqueles com 1-9 dentes e edentados, maior entre pardos/negros/índios, que nunca usaram serviços, que apresentavam alterações de tecidos moles, que relataram pouca e média/muita dor, que auto-avaliaram sua aparência e mastigação como regular ou ruim/péssima, entre os que relataram seus relacionamentos sociais pouco ou muito afetados pelas condições bucais e entre os que avaliavam necessitar de tratamento bucal. Apesar das precárias condições bucais, a maioria auto-avaliou positivamente sua saúde bucal. Condições subjetivas estiveram mais fortemente associadas do que as objetivas. Os resultados sugerem iniqüidade e permitem orientar políticas públicas que objetivem saúde bucal e qualidade de vida.

Saúde Bucal; Idoso; Qualidade de Vida

\section{Colaboradores}

A. M. E. B. L. Martins idealizou o trabalho, realizou as análises estatísticas e a revisão da literatura, escreveu a primeira versão do manuscrito e participou de sua revisão. S. M. Barreto trabalhou na concepção, redação, revisão da literatura, análise estatística e revisão do manuscrito. I. A. Pordeus contribuiu na revisão da literatura, redação e revisão do manuscrito.

\section{Agradecimentos}

Agradecemos à Coordenação de Saúde Bucal do Ministério da Saúde e à equipe de campo do inquérito, bem como aos participantes do estudo. A. M. E. B. L. Martins recebeu apoio logístico das Faculdades Unidas do Norte de Minas (FUNORTE/SOEBRAS) durante a realização deste trabalho. S. M. Barreto e I. A. Pordeus são bolsistas de produtividade em pesquisa do Conselho Nacional de Desenvolvimento Científico e Tecnológico (CNPq). 


\section{Referências}

1. Gilbert L. Social factors and self-assessed oral health in South Africa. Community Dent Oral Epidemiol 1994; 22:47-51.

2. Benyamini $Y$, Leventhal $H$, Leventahal EA. Self rated oral health as an independent predictor of self rated general health, self esteem and life satisfaction. Soc Sci Med 2004; 59:1109-16.

3. Silva SRC, Fernandes RAC. Autopercepção das condições de saúde bucal por idosos. Rev Saúde Pública 2001; 35:349-55.

4. Locker D. Clinical correlates of change in self perceived oral health in older adults. Community Dent Oral Epidemiol 1997; 25:199-203.

5. Reis SCGB, Marcelo VC. Saúde bucal na velhice: percepção dos idosos, Goiânia, 2005. Ciênc Saúde Coletiva 2006; 11:191-9.

6. Reisine ST, Bailit HL. Clinical oral health status and adult perceptions of oral health. Soc Sci Med 1980; 14:597-605.

7. Mathias RE, Atchison KA, Lubben JE, De-Jong F, Schweitzer SO. Factors affecting self-ratings of oral health. J Public Health Dent 1995; 55:197-204.

8. Atchison KA, Gift HC. Perceived oral health in a diverse sample. Adv Dent Res 1997; 11:272-80.

9. Gift HC, Atchison KA, Drury TF. Perceptions of the natural dentition in the context of multiple variables. J Dent Res 1998; 77:1529-38.

10. Locker D, Clarke M, Payne B. Self perceived oral health status, psychological well-being, and life satisfaction in an older population. J Dent Res 2000; 79:970-5.

11. Heft MW, Gilbert GH, Shelton BJ, Duncan RP. Relationship of dental status, sociodemographic status, and oral symptoms to perceived need for dental care. Community Dent Oral Epidemiol 2003; 31:351-60.

12. Kiyak HA. Age and culture: influences on ora health behavior. Int Dent J 1993; 43:9-16.

13. Borrel LN, Taylor GW, Borgnakke WS, Wollfolk MW, Nyquist LV. Perception of general and oral health in white and African American adults: assessing the effect of neighborhood socioeconomic conditions. Community Dent Oral Epidemiol 2004; 32:363-73.

14. Coordenação Nacional de Saúde Bucal, Departamento de Atenção Básica, Secretaria de Atenção à Saúde, Ministério da Saúde. Projeto SB BRASIL 2003: condições de saúde bucal da população brasileira 2002-2003. Resultados principais. Brasília: Ministério da Saúde; 2004.

15. Martins AMEBL, Barreto SM, Pordeus IA. Uso de serviços odontológicos entre idosos brasileiros. Rev Panam Salud Pública 2007; 22:425-30.

16. World Health Organization. Oral heath surveys: basic methods. $4^{\text {th }}$ Ed. Geneva: World Heath Organization; 1997.

17. Área Técnica de Saúde Bucal, Departamento de Atenção Básica, Secretaria de Políticas da Saúde, Ministério da Saúde. Projeto SB 2000: condições de saúde bucal da população brasileira no ano 2000. Brasília: Ministério da Saúde; 2000.
18. Roncalli AG, Frazão P, Pattussi MP, Araújo IC, Ely HC, Batista SM. Projeto SB 2000: uma perspectiva para a consolidação da epidemiologia em saúde bucal coletiva. Revista Brasileira de Odontologia em Saúde Coletiva 2000; 1:9-25.

19. Locker D, Gibson B. Discrepancies between selfratings of and satisfaction with oral health in two older adult populations. Community Dent Oral Epidemiol 2005; 33:280-8.

20. Pestana MH, Gageiro JN. Análise de dados para ciências sociais. A complementaridade do SPSS. $3 a$ Ed. Lisboa: Edições Silabo; 2003.

21. Kirkwood BR, Sterne JAC. Medical statistics. 2nd Ed. Malden: Blackwell Science Ltd.; 2003.

22. Nunes CIP, Abegg C. Factors associated with oral health perception in older Brazilians. Gerodontology 2008; 25:42-8.

23. Petersen PE. Dental visits and self-assessment of dental health in the adult Danish population. Community Dent Oral Epidemiol 1983; 11:162-8.

24. Murtomaa H, Laine P. Differentiating dental satisfaction in Finns by means of discriminant analyses. Community Dent Oral Epidemiol 1985; 13:7-10.

25. Atchison KA, Mathias RE, Dolan TA, Lubben JE, Jong FD, Mayer-Oakes SA. Comparison of oral health ratings by dentists and dentate elders. J Public Health Dent 1993; 53:223-30.

26. Kressin NR. Associations among different assessments of oral health outcomes. J Dent Educ 1996; 60:501-7.

27. Kressin NR, Atchison KA, Miller D. Comparing the impact of oral disease in two populations of older adults: application of the geriatric oral health assessment index. J Public Health Dent 1997; 57: 224-32.

28. Lang WP, Borgnakke WS, Taylor GW, Woolfolk MW Ronis DL, Nyquist LV. Evaluation and use of an index of oral health status. J Public Health Dent 1997; 57:233-42.

29. Federation Dentaire Internationale. Global goals for oral health in the year 2000. Int Dent J 1982; 32:74-7.

30. Moreira RS, Nico LS, Tomita NE, Ruiz T. A saúde bucal do idoso brasileiro: revisão sistemática sobre o quadro epidemiológico e acesso aos serviços de saúde bucal. Cad Saúde Pública 2005; 21:1665-75.

31. Jokovik A, Locker D. Dissatisfaction with oral health status in an older adult population. J Public Health Dent 1997; 57:40-7.

32. Locker D, Slade G. Association between clinical and subjective indicators of oral health status in an older adult population. Gerodontology 1994; 11:108-14.

33. Cruz ICF. Saúde e iniqüidades raciais no Brasil: o caso da população negra. Online Braz J Nurs (Online) 2006; 5(2). http://www.uff.br/objnursing/ index.php/nursing/article/view/400/95.

34. Matos DL, Lima-Costa MF. Auto-avaliação da saúde bucal entre adultos e idosos residentes na Região Sudeste: resultados do Projeto SB-Brasil, 2003. Cad Saúde Pública 2006; 22:1699-707. 
35. Donaldson AC, Riggio MP, Rolph HJ, Bagg J, Hodge PJ. Clinical examination of subjects with halitosis. Oral Dis 2007; 13:63-70.

36. Bromley SM. Smell and taste disorders: a primary care approach. Am Fam Phys 2000; 61:427-36.

37. Locker D, MillerY. Subjectively reported oral health status in a adult population. Community Dent Oral Epidemiol 1994; 22:425-30.

38. Martins AMEBL, Barreto SM, Pordeus IA. Fatores relacionados à autopercepção da necessidade de tratamento odontológico entre idosos. Rev Saúde Pública 2008; 42:487-96.
39. Elderton RJ. Ciclo restaurador repetitivo. In: Kriger L, coordenador. ABOPREV. Promoção de saúde bucal: paradigma, ciência, humanização. 3a Ed. São Paulo: Editora Artes Médicas; 2003. p. 194-249.

40. Matos DL, Giatti L, Lima-Costa MF. Fatores sócio-demográficos associados ao uso de serviços odontológicos entre idosos brasileiros: um estudo baseado na Pesquisa Nacional por Amostra de Domicílios. Cad Saúde Pública 2004; 20:1290-7.

Recebido em 05/Mar/2008

Versão final reapresentada em 15/Jul/2008

Aprovado em 05/Ago/2008 